\title{
Experimental Study on the Heterogeneity of Mechanical Properties of Friction Stir Welded Joints with the Digital Image Correlation Method
}

\author{
H. Li, Q.-L. Dai, and Q.-Y. Shi ${ }^{1}$
}

State Key Laboratory of Tribology, Department of Mechanical Engineering, Tsinghua University, Beijing, China

${ }^{1}$ shqy@mail.tsinghua.edu.cn

The heterogeneity of mechanical properties of friction stir welded 6082 aluminum butt joints were analyzed with the digital of image correlation (DIC) method. The DIC method provides instantaneous full-field strain distribution data for weld surfaces. Visualized strain field images and local stress-strain curves of weld subzones were derived from these data. The heterogeneity was evaluated by assessing strain concentrations in the strain field images and comparing the constitutive parameters of weld subzones. Furthermore, by comparing these variables of different surfaces of the weld, the potential impact of the mutual constraint between subzone materials on the heterogeneity level was analyzed..

Keywords: friction stir welding, heterogeneity, digital image correlation.

Introduction. Friction stir welding (FSW) is a solid state joining technique, which was invented at the Welding Institute (TWI) of UK in 1991 [1]. This technique creates flawless welds of aluminum alloy joints, including certain series of high-strength alloys that used to be considered non-weldable [2-4]. The FSW technique is widely used in the manufacturing, and especially in the transportation industry. During the welding process, material in the joint experiences intense plastic deformation due to high temperatures, which results in generation of three distinct zones in the weld [5]. Those zones are: (i) the nugget, which is the center of the weld and is constituted by small recrystallized equiaxed grains; (ii) the thermo-mechanically affected zone (TMAZ), which is around the nugget and adjacent to it and consists of deformed needle-shaped grains; and (iii) the heat-affected zone (HAZ), which is next to the TMAZ and consists of larger grains than those of the base material (BM). If the base material is also taken into account, a typical FSW weld consists of the four parts, as is shown in Fig. 1. Noteworthy is that specimens in this study have very narrow TMAZ zone between the nugget and HAZ, which can be revealed only with the help of a microscope.

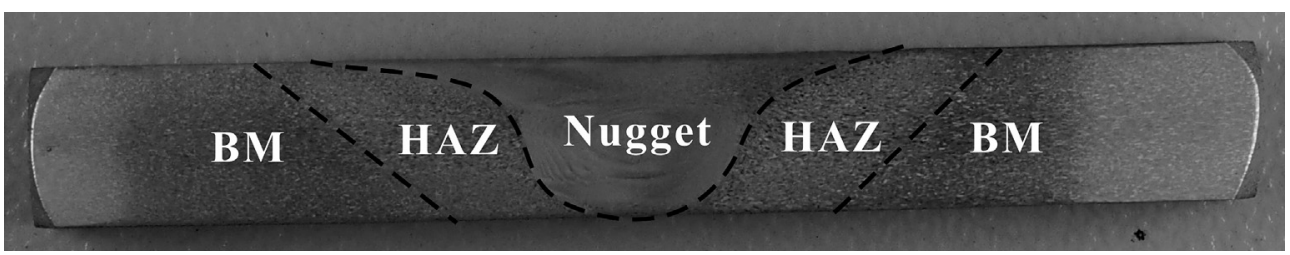

Fig. 1. Cross section of 6082 aluminum alloy FSW joint.

The variation in subzone metallurgical structure causes the variation in subzone mechanical properties. While the nugget strength can compete with that of the base material, the HAZ strength may be much less. This heterogeneity in subzone mechanical properties constrains the strength of the total joint. The aim of this study is to clarify what improvements are needed to increase the strength of the entire joint. However, measuring the local mechanical properties of subzones with a high accuracy is yet quite problematic. 
The technique that is commonly used to analyze the mechanical characterization of welds is the microhardness testing [6], which indirectly derives the constitutive parameters of material mainly based on engineering experience [7]. Other techniques, such as using extensometers or cutting miniature specimens from the target zone, can provide local stress-strain data with a high-quality resolution. Based on the digital image processing and numerical computing techniques, the digital image correlation (DIC) method analyzes digital images taken from the specimen surface that is undergoing deformation and directly provides the instantaneous full field strain data [8-10], from which the strain field image and local constitutive parameters can be extracted at a high resolution.

Another aim of this study was to evaluate the degree of heterogeneity of the mechanical properties of the Al 6082 FSW weld and to assess whether the weld subzones deform separately or uniformly under an uniaxial tensile force. In this study, digital images of weld specimens under tensile tests were processed using the DIC method. From the DIC results, a series of strain field images were extracted to reveal the deformation evolution of the joint. Local stress-strain curves were constructed and local constitutive parameters were measured. In addition, significant differences between heterogeneity levels that appeared on various surfaces of the weld led to further analysis of the mutual constraints of subzone material that could partly neutralize such heterogeneity when the weld was deformed as a whole.

1. Experimental Procedure. Butt welds created by FSW on $5 \mathrm{~mm}$ thick plates are tested. The base material is 6082 aluminum alloy, which is widely used in the manufacturing of high-speed train carriages. The FSW parameters are shown in Table 1. This set of parameters was utilized in our earlier experiments; therefore, the ability to obtain robust and flawless joint welds was already established.

$\mathrm{T}$ a b 1 e 1

FSW Parameters

\begin{tabular}{|c|c|c|c|c|c||}
\hline $\begin{array}{c}\text { Plate thickness } \\
(\mathrm{mm})\end{array}$ & $\begin{array}{c}\text { Pin length } \\
(\mathrm{mm})\end{array}$ & $\begin{array}{c}\text { Pin diameter } \\
(\mathrm{mm})\end{array}$ & $\begin{array}{c}\text { Shoulder } \\
\text { diameter }(\mathrm{mm})\end{array}$ & $\begin{array}{c}\text { Rotational } \\
\text { speed }(\mathrm{rpm})\end{array}$ & $\begin{array}{c}\text { Welding speed } \\
(\mathrm{mm} / \mathrm{min})\end{array}$ \\
\hline 5 & 4.7 & 4 & 20 & 1800 & 200 \\
\hline
\end{tabular}

After the joint weld was made, three longitudinal specimens were taken from the welded plate, and an additional specimen was taken from an unwelded 6082 plate. The configuration and size of the specimens, which are shown in Fig. 2, were compliant to the ISO 4136-2001 standards, and a coordinate system was established defining the specimen's length as $y$-direction, width as $x$-direction, and thickness as $z$-direction. The welded specimen upper and lower surfaces were milled on a plane, ensuring a uniform thickness. The tensile tests were performed under room temperature conditions using a $30 \mathrm{kN}$ universal testing machine with a $2 \mathrm{~mm} / \mathrm{min}$ tensile load rate.

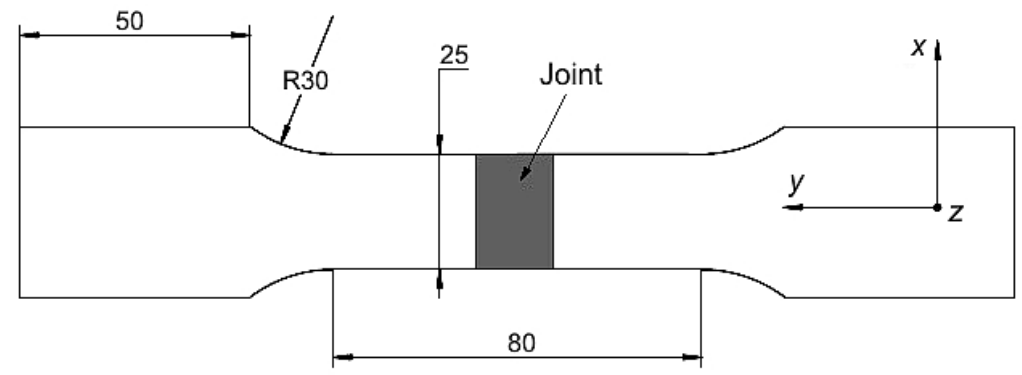

Fig. 2. Specimen design. 
The DIC procedure requires a random speckled pattern to be sprayed onto the specimen target surface before the tensile test. For the purpose of raising the DIC analysis accuracy, the black speckled pattern must uniformly cover the white background, devoid of losing its randomness, as shown in the upper part of Fig. 3. A charge-coupled device (CCD) camera mounted on a tripod was placed in front of the specimen, and its position was carefully adjusted so that the axis of its lens was perpendicular to the specimen surface. During the tensile test, this camera produced two images of the specimen surface per second, and all of these images were saved with time information in their file name. At the beginning of each test, certain measures were taken to ensure that operation of the universal testing machine and the camera started simultaneously, thus the stress and strain data could be matched with the minimal error in time. After the tensile tests, the images were processed by a professional DIC software package (VIC2D), which provided field displacement and strain data for each image. The lower section of Fig. 3 shows a processed image representing the instantaneous strain field on the back of the specimen surface at $0.5 \mathrm{~s}$ before it cracked.

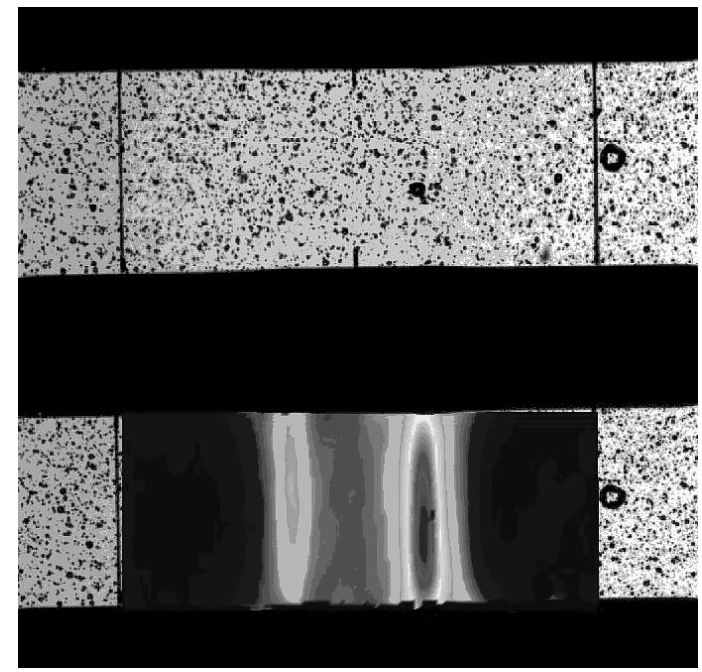

Fig. 3. Speckled pattern and DIC result.

Since the FSW joint geometry is quite complex, the DIC results of one surface do not represent the strain distribution pattern of the total joint. Around the tensile force axis, there were four surfaces for each specimen. Considering that two side surfaces of the joint cross section are symmetrical, only three surfaces need to be analyzed. The most reliable procedure to analyze three different directions of a specimen would be simultaneous capturing of images of the specimen three surfaces. However, due to the limitations of having just a single camera available, the three surfaces could only be analyzed from the tensile tests. Therefore, three specimens were sprayed on different surfaces. The first specimen was the front surface, into which the tool pin was plunged and was perpendicular to the $z$ axis. The second specimen was the back surface, which was adjacent to the back plate and was perpendicular to the $z$ axis. The third specimen was the side surface, which was the cross section of the joint and was perpendicular to the $x$ axis. Thus, the entire FSW joint was analyzed, and strain fields in three directions were observed. To ensure that three DIC results were comparable and reliable enough to represent the identical joint condition, the camera installation and DIC parameters remained unchanged during the three tests.

Since the DIC method does not provide stress data for constructing the local stress-strain curves, while no methods for measuring the instantaneous local stress are 
currently available, therefore the local stress data were replaced with the uniform stress data obtained using the universal testing machine. Researchers $[11,12]$ have certified the accuracy of this method by comparing the $0.2 \%$ yield stress obtained by the DIC and by extensometer, which indicated that these two results differed less than by $4 \%$.

2. Results and Discussion. Figure 4 depicts the local stress-strain curves from different subzones of the lower surface. Strain concentration of this surface has the best correlation with the subzone location. Five testing points were selected along the center axis of the specimen, representing $\mathrm{BM} / \mathrm{HAZ}$ on the advancing side (AS), and nugget, $\mathrm{BM} / \mathrm{HAZ}$ on the retreating side (RS). The TMAZ was not tested because the limited CCD resolution made it impossible to accurately identify this subzone in the images. Moreover, in the strain field images, this subzone did not demonstrate a clear contour, but appeared as a narrow transitional area between the nugget and HAZ.

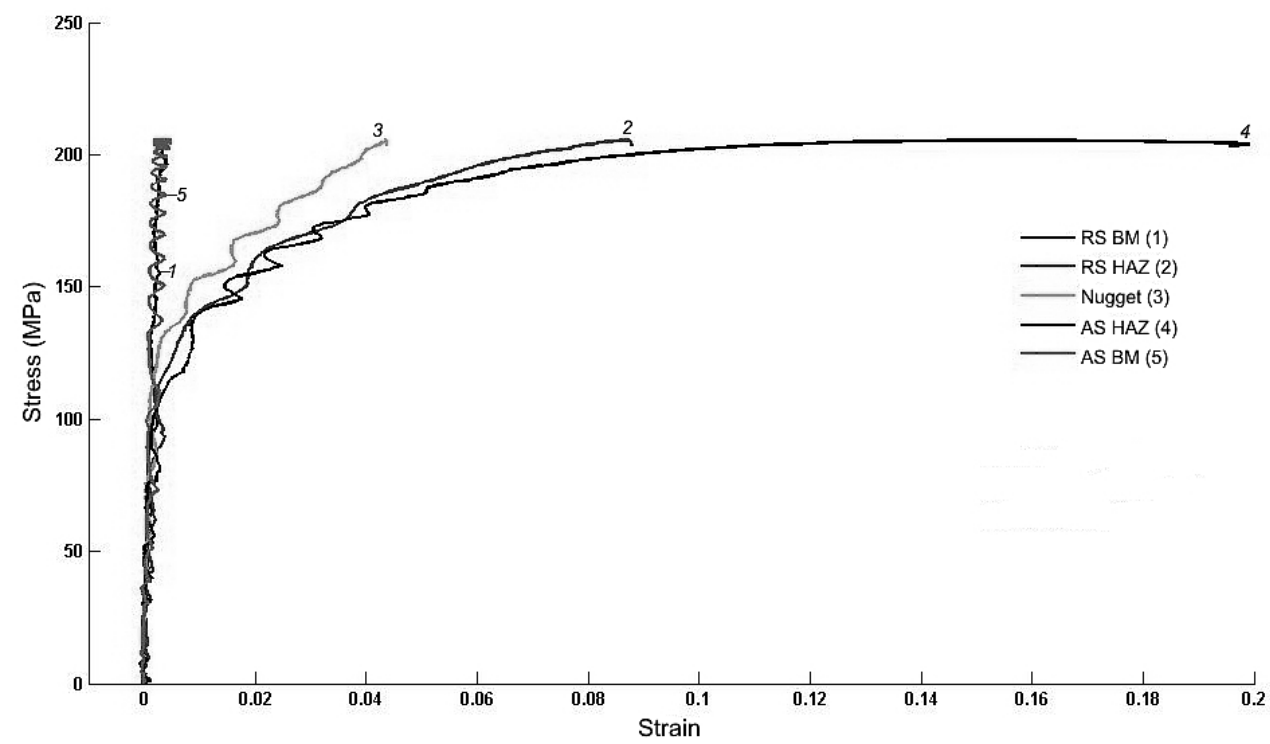

Fig. 4. Local stress-strain curves of the specimen lower surface on the advancing side (AS) and retreating side $(\mathrm{RS})$.

In Fig. 4, there are two straight lines that manifest a monotonic increase, which represents the deformation process of AS BM and RS BM. This proves that the material in $\mathrm{BM}$ did not yield until it cracked. The $0.2 \%$ yield stresses of both HAZs are nearly identical (about $120 \mathrm{MPa}$ ). The ultimate strength of AS HAZ is approximately $200 \mathrm{MPa}$; however, because no actual failure happened in this area, the ultimate strength of RS HAZ could not be accurately measured from the plot in Fig. 4. However, a slight difference between the curves of the two HAZs implies a small difference between the respective two ultimate strengths. This result indicates the behavior earlier observed via the strain measurements, which initially showed simultaneous strain concentrations in the two HAZs that ultimately increased due to their competition. The RS HAZ eventually stopped growing. This competitive behavior is shown in Fig. 5. The similarity between the stress-strain curves of the two HAZs suggests that the significant difference in the densities of the two HAZ strain concentration belts can be attributed not to any significant difference in their mechanical properties, but to the fact that necking happened first in the AS HAZ.

The local mechanical properties of different zones of the joint are shown in Table 2 . The data in Table 2 show that the $0.2 \%$ yield stress of HAZ dropped to $41 \%$ of BM, the ultimate strength dropped to $63 \%$ of $\mathrm{BM}$, while elongation remained the same. 
$\mathrm{T}$ a b 1 e 2

Local Mechanical Properties of Al 6082 FSW Joint (Lower Surface)

\begin{tabular}{|c|c|c|c|}
\hline Zone & $\begin{array}{c}0.2 \% \text { yield stress } \\
(\mathrm{MPa})\end{array}$ & $\begin{array}{c}\text { The ultimate strength } \\
(\mathrm{MPa})\end{array}$ & $\begin{array}{c}\text { Elongation } \\
(\%)\end{array}$ \\
\hline BM & 290 & 320 & 19 \\
\hline Nugget & 135 & - & - \\
\hline RS HAZ & 120 & 200 & 20 \\
\hline
\end{tabular}

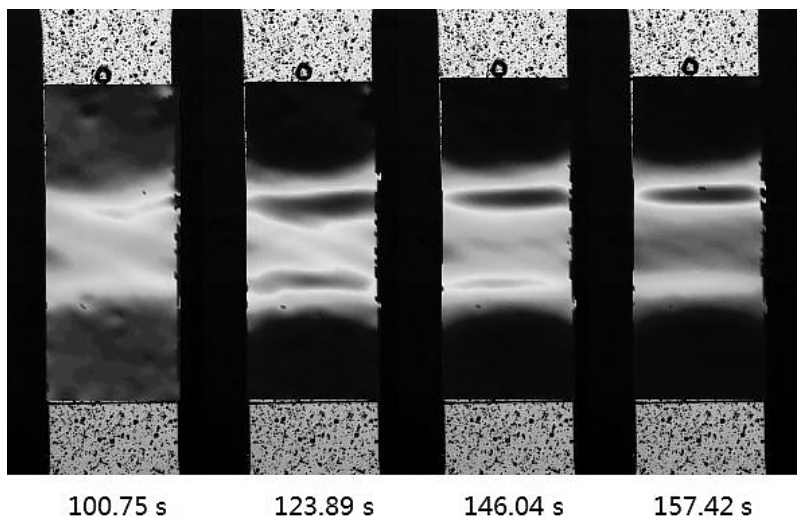

Fig. 5. Strain growth competition between AS HAZ and RS HAZ.

The constitutive parameters obtained from the upper surface are given in Table 2 with small discrepancy/error, yet the data extracted from the side surface are significantly different. The local stress-strain curves of the side surface are shown in Fig. 6: although the latter curves are quite coarse because of the limited width of the side surface restricting the DIC resolution, basic trends could still be observed and parameters could be estimated with a sufficient accuracy. Curves of AS HAZ, nugget, and RS HAZ almost coincide, while the $0.2 \%$ yield stress of these subzones vary slightly around $150 \mathrm{MPa}$. As compared to the upper and lower surfaces, the mechanical properties of the side surface are more uniformly distributed.

Strain field images of the three surfaces are compared in Fig. 7, which shows their differences more clearly. These images represent the strain distribution at $0.5 \mathrm{~s}$ before cracking begins. The upper and lower surfaces demonstrate a concentrated large strain belt (the maximum value is 0.232 ) at the AS HAZ and a strain concentration belt with less intensity at the RS HAZ. This type of concentration is less obvious at the side surface, which represents the inner part of the weld. The highest strain (about 0.07) observed on the side surface is much lower than those on the upper and lower surfaces. The joint mechanical properties manifest a significant heterogeneity of the upper and lower surfaces, but show a relatively mild homogeneity of the side surface. Additionally, the absence of a pronounced area on the edge of the side surface is observed, which means that the large strain observed in the other two surfaces may be constrained within a very thin layer on and below the surface. This phenomenon may be caused by the mutual restraint between the adjacent materials belonging to different subzones. The adjacent materials with different mechanical properties restrain each other and are deformed following their own constitutive equations without affecting the adjacent material deformation. Thus, they cooperate with each other and demonstrate common properties as a whole. At the upper and lower surfaces, the HAZs are located in the belt area perpendicular to the tensile force. The 


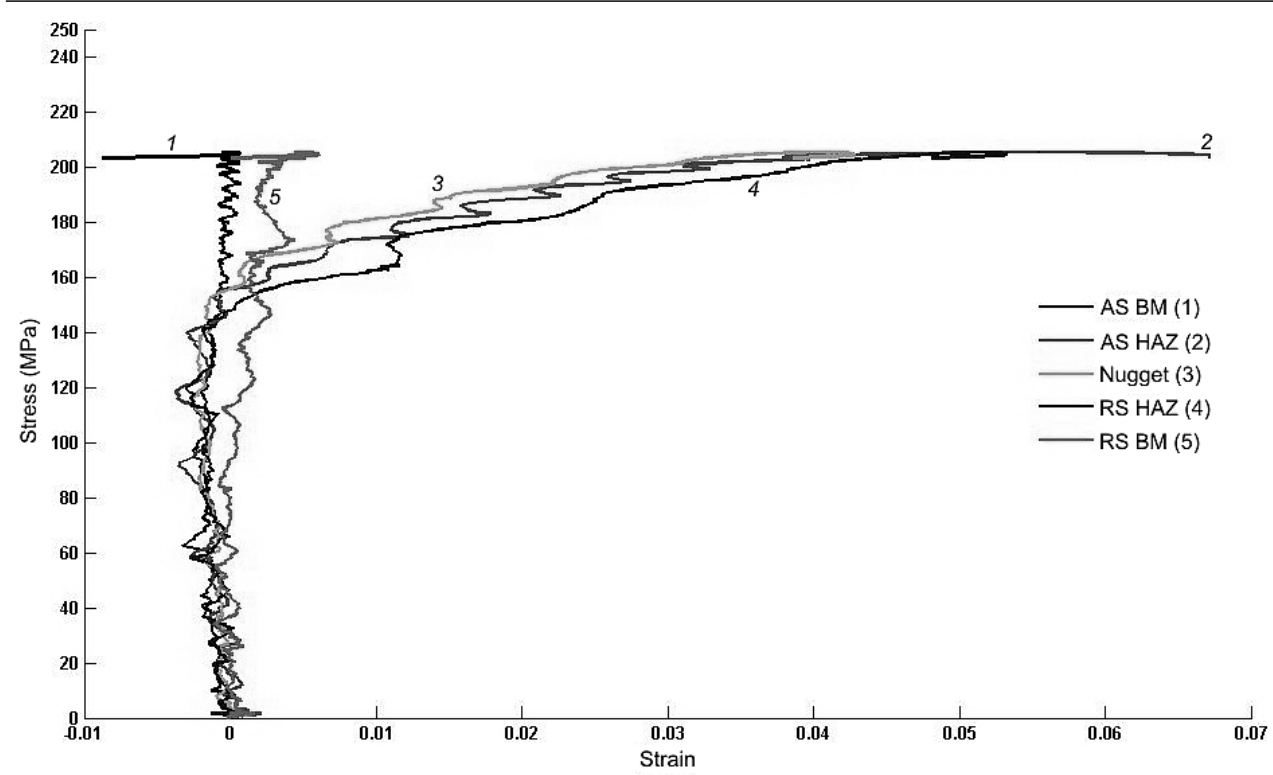

Fig. 6. Local stress-strain curves of the side surface.

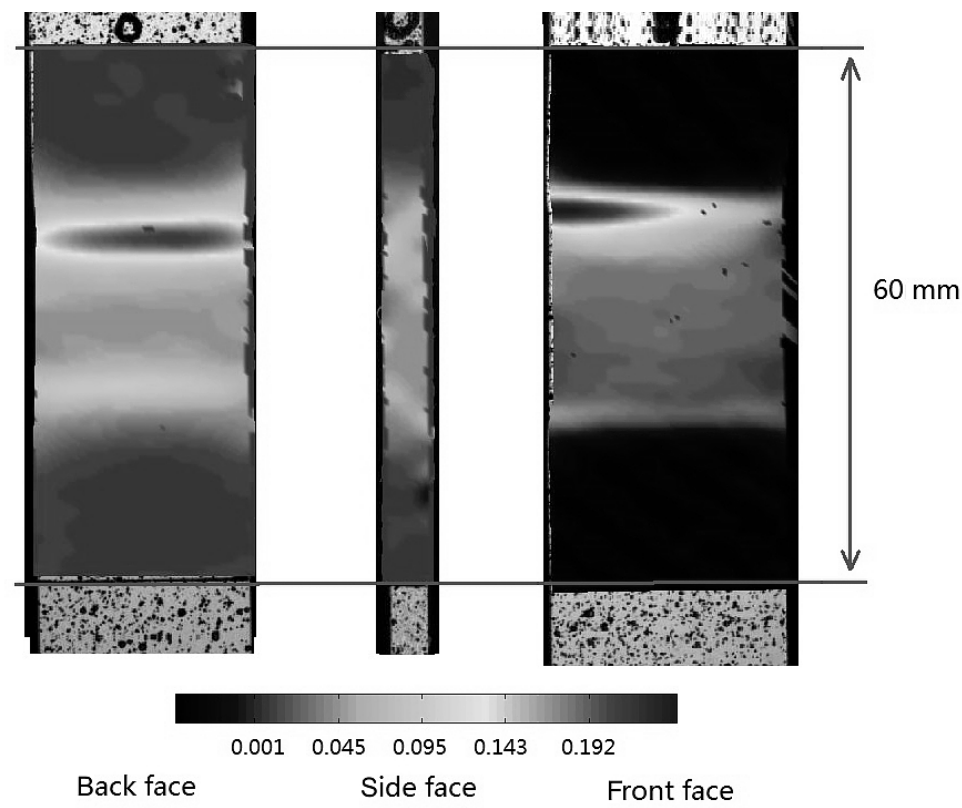

Fig. 7. Strain fields of different surfaces at $0.5 \mathrm{~s}$ before cracking.

deformation in the tensile direction of these HAZs does not affect the adjacent materials in the BM or nugget, while the HAZ belts at the side surface have a nonrectangular angle with the tensile direction. Therefore, HAZ material deformation is partially restrained by the adjacent BM or nugget material. The mutual restraint assumption may imply the following two possibilities. Firstly, if a complex-shaped HAZ geometry can be achieved, this will result in a higher homogeneity of its mechanical properties and, thus, in the improvement of the joint strength. Secondly, a higher homogeneity may be attained by strengthening the surface material. However, these two possibilities require a further verification. 
Conclusions. The results of this work provide the full field strain distribution and local constitutive parameters of Al $6082 \mathrm{FSW}$ welds. The mechanical property heterogeneity was evaluated both qualitatively and quantitatively. It was concluded that the weld showed obvious heterogeneity in its local mechanical properties.

1. The ultimate strength of HAZ was below the yield strength of BM, while the yield strength of $\mathrm{HAZ}$ was $11 \%$ below that of the nugget.

2. The reason for more uniform deformation of the inner part of the weld than that in the surface layers during the same tensile process can be reduced to the fact that the mutual constraints of subzone material may partly neutralize the heterogeneity of the mechanical properties when the weld is deformed as a whole.

1. W. M. Thomas, E. D. Nicholas, J. C. Needham, et al., Friction Stir Butt Welding, International Patent Application No. PCT/GB92/02203 and GB Patent Application No. 9125978.8, 6 December 1991.

2. C. J. Dawes and W. M. Thomas, "Friction stir process welds aluminum alloys," Weld. J., 75, No. 3, 41-45 (1996).

3. E. A. El-Danaf, M. M. El-Rayes, and M. S. Soliman, "Friction stir processing: An effective technique to refine grain structure and enhance ductility," Mater. Design, 31, 1231-1236 (2010).

4. R. Nandan, T. DebRoy, and H. K. D. H. Bhadeshia, "Recent advances in friction stir welding - process, weldment structure and properties," Progr. Mater. Sci., 53, 980-1023 (2008).

5. R. S. Mishra and Z.-Y. Ma, "Friction stir welding and processing," Mater. Sci. Eng. $R, \mathbf{5 0}, 1-78$ (2005).

6. C. Leitão, I. Galvão, R. M. Leal, and D. M. Rodrigues, "Determination of local constitutive properties of aluminium friction stir welds using digital image correlation," Mater. Design, 33, 69-74 (2012).

7. Y.-L. Song, L. Hua, D.-N. Chu, and J. Lan, "Characterization of the inhomogeneous constitutive properties of laser welding beams by the micro-Vickers hardness test and the rule of mixture," Mater. Design, 37, 19-27 (2012).

8. B. Pan, K.-M. Qian, H.-M. Xie, and A. Asundi, "Two-dimensional digital image correlation for in-plane displacement and strain measurement: a review," Measur. Sci. Technol., 20, No. 6, 1-17 (2009).

9. T.-C. Chu, W. F. Ranson, and M. A. Sutton, "Applications of digital-image-correlation techniques to experimental mechanics," Exp. Mech., 25, No. 3, 232-244 (1985).

10. G. Vendroux and W. G. Knauss, "Submicron deformation field measurements: Part 2. Improved digital image correlation,” Exp. Mech., 38, No. 2, 86-92 (1998).

11. H. J. K. Lemmen, R. C. Alderliesten, R. Benedictus, et al., "The power of digital image correlation for detailed elastic-plastic strain measurements," in: WSEAS Int. Conf. on Engineering Mechanics, Structures, Engineering Geology (EMESEG'08, July 22-24, 2008, Heraklion, Crete Island, Greece), pp. 73-89.

12. C. Hang, G. Yang, Y.-L. Li, et al., "Application of digital image correlation method to test of mechanical properties for weld materials," Acta Aeronaut. Astronaut. Sinica, 34, No. 10, 2372-2382 (2013). 